\title{
STRUCTURE OF HEREDITARY ORDERS ${ }^{1}$
}

\author{
BY ARMAND BRUMER
}

Communicated by Daniel Zelinsky, May 9, 1963

Maximal orders over Dedekind domains have long played an important role in arithmetic $[4 ; 6]$. Recently Auslander and Goldman [1] have introduced the concept of hereditary order, which generalizes that of maximal order and which is more satisfactory for many purposes. An $R$-order $\Lambda$ in a central simple algebra over the quotient field of the Dedekind domain $R$ is said to be an hereditary $R$-order if the global dimension of $\Lambda$ is one [ 3 ].

The aim of this note is to announce a fairly complete description of hereditary orders. ${ }^{2}$ The proofs and applications will be given elsewhere.

Reminder. All modules are finitely generated and unitary. Algebras are finite dimensional over their centers.

1. Local theory. Throughout this section, $R$ denotes a discrete rank one valuation ring with field of quotients $K ; D$ is a central division algebra over $K$ and $V$ is a right $D$-module. Let $\Omega$ be a maximal $R$-order in $D$ and let $\mathfrak{M}$ be the unique maximal two-sided ideal of $\Omega$.

An $\Omega$-chain $\varepsilon=\left\{E_{k}\right\}$ in $V$ of period $r$ is a decreasing sequence of subgroups of $V$ satisfying the following axioms for all integers $k$ :

(i) $E_{k}$ is a right $\Omega$-module;

(ii) $E_{k+1}$ is a proper $\Omega$-submodule of $E_{k}$;

(iii) $E_{k+r}=E_{k} \mathfrak{M}$.

We may, of course, define homomorphisms of $\Omega$-chains in the obvious manner. If $\varepsilon$ and $\mathcal{F}$ are $\Omega$-chains of period $r$ in $V$, then

$$
\operatorname{Hom}_{\Omega}(\varepsilon, F)=\left\{f \in \operatorname{Hom}_{D}(V, V) \mid f\left(E_{k}\right) \subset F_{k} \text { for all integers } k\right\} \text {. }
$$

The following gives a concrete representation for hereditary orders in central simple algebras, if we recall Wedderburn's theorem.

TheOREM 1. An R-order $\Lambda$ in the central simple algebra $\operatorname{Hom}_{D}(V, V)$ is an hereditary $R$-order if and only if there is an $\Omega$-chain $\varepsilon$ in $V$ such that $\Lambda=\operatorname{Hom}_{\Omega}(\varepsilon, \varepsilon)$. Then $\varepsilon$ is the set of all $(\Lambda, \Omega)$-modules in $V$.

1 This note is a summary of a dissertation being submitted in partial fulfillment of the requirements for the $\mathrm{Ph}$.D. at Princeton University. I would like to express my gratitude to Professor Maurice Auslander for suggesting this study and for many valuable discussions.

2 Special cases of our results have been obtained independently by Dr. Manabu Harada. 
REMARK. The period of $\varepsilon$ is equal to the number of maximal two sided ideals of $\Lambda=\operatorname{Hom}_{\Omega}(\varepsilon, \varepsilon)$, and to the number of maximal $R$ orders which contain $\Lambda$. The latter may be given explicity as follows: if $\varepsilon=\left\{E_{k}\right\}$, and $\Gamma_{k}=\operatorname{Hom}_{\Omega}\left(E_{k}, E_{k}\right)$ then $\Gamma_{1}, \Gamma_{2}, \cdots, \Gamma_{r}$ are the maximal $R$-orders which contain $\Lambda$.

The proof of Theorem 1 gives as a bonus the structure of projective modules over an hereditary order $\Lambda$, i.e., of $\Lambda$-modules which are $R$ torsion free [1, Proposition 2.8].

THEOREM 2. Let $\Lambda$ be an hereditary R-order in $\operatorname{Hom}_{D}(V, V)$. Let $\mathcal{E}=\left\{E_{k}\right\}$ be the $\Omega$-chain of $(\Lambda, \Omega)$-modules in $V$ and suppose $\mathcal{E}$ is of period $r$. If $F$ is a projective left $\Lambda$-module, then $F \cong \oplus_{k=1}^{r} s_{k} E_{k}$, where $s_{k} E_{k}$ stand for the direct sum of $s_{k}$ copies of $E_{k}$. The integers $s_{1}, \cdots, s_{r}$ are uniquely determined by the $\Lambda$-module $F$. In particular $E_{1}, \cdots, E_{r}$ are all the indecomposable projective left $\Lambda$-modules.

Let $\Sigma$ be a central simple algebra over $K$. Let $\hat{K}$ be the completion of $K$, then $\hat{\Sigma}=\hat{K} \otimes_{K} \Sigma$ is a central simple algebra and thus is the algebra of $m \times m$-matrices over a suitable division algebra. We call $m$ the complete degree of $\boldsymbol{\Sigma}$.

Suppose $\Lambda$ is an hereditary $R$-order in the central simple algebra $\Sigma=\operatorname{Hom}_{D}(V, V)$ and suppose that $\varepsilon=\left\{E_{k}\right\}$, the $\Omega$-chain of $(\Lambda, \Omega)$ modules in $V$, has period $r$. If $\Omega$ is a maximal $R$-order in $D$ and $\mathfrak{M}$ is its maximal two-sided ideal, then $\Omega / \mathfrak{M}$ is a simple algebra, so that all simple $\Omega / \mathfrak{M}$ modules are isomorphic. One sees easily that $E_{1} / E_{1} \mathfrak{M}$ is the direct sum of $m$ simple $\Omega / \mathfrak{M}$-modules, where $m$ is the complete degree of $\Sigma$. Finally, let $n_{k}$ be the number of components in a direct sum decomposition of $E_{k} / E_{k+1}$ into simple $\Omega / \mathfrak{M}$-modules. Then $n_{1}+\cdots+n_{r}=m$ and $\Lambda$ determines the partition $\left(n_{1}, \cdots, n_{r}\right)$ of $m$ uniquely up to cyclic permutations. We consider two partitions of the integer $m$ to be equivalent if they differ by a cyclic permutation. Let $P(m)$ be the set of equivalence classes of partitions of $m$ and let $H(\boldsymbol{\Sigma})$ be the set of isomorphism classes of hereditary $R$-orders in $\boldsymbol{\Sigma}$. Then the above gives a map $I: H(\Sigma) \rightarrow P(m)$ called the invariant of hereditary R-orders. Explicitly $I(\Lambda)=\left(n_{1}, \cdots, n_{r}\right)$ in the notation above. The following theorem classifies hereditary $R$-orders completely.

Theorem 3. Let $\Sigma$ be a central simple algebra over $K$. Suppose that the complete degree of $\Sigma$ is $m$. Then the invariant sets up a 1-1 correspondence between $H(\Sigma)$ and $P(m)$.

REMARKs. (1) Roughly speaking the above theorem gives complete numerical invariants to hereditary orders. 
(2) Let $\Lambda$ be an hereditary $R$-order and let $\Re$ be its radical. If $I(\Lambda)=\left(n_{1}, \cdots, n_{r}\right)$, then $\Re$ is a principal ideal if and only if $n_{1}=\cdots=n_{r}$.

(3) This result may be used to solve a problem stated in [2, pp. 394-395].

2. Global results. It is easy to show that local hereditary orders may be pasted together to give global hereditary orders. More precisely:

Theorem 4. Let $R$ be a Dedekind domain with field of quotients $K$ and let $\Sigma$ be a central simple algebra over $K$. Let $\Gamma$ be a maximal $R$-order in $\Sigma$. Then:

(i) If $\Lambda$ is an hereditary $R$-order in $\Sigma$, then $\Lambda_{p}=\Lambda \otimes_{R} R_{p}$ is an hereditary $R_{p}$-order for all maximal ideals $p$ of $R$. Also $\Lambda_{p}=\Gamma_{p}$ for almost all $p$.

(ii) Given any collection $\{\Lambda(p)\}_{p}$ of subrings of $\Sigma$ such that $\Lambda(p)$ $=\Gamma_{p}$ for almost all $p$ and such that $\Lambda(p)$ is an hereditary $R_{p}$-order for all maximal ideals $p$ of $R$. Then there is a unique hereditary $R$-order $\Lambda$ such that $\Lambda_{p}=\Lambda(p)$ for all $p$.

The following global version of Theorem 2 was suggested by a special case proved in [5].

Theorem 5. Let $R$ be a Dedekind domain with field of quotients $K$ and let $\Sigma$ be a central simple algebra over $K$. Suppose $\Lambda$ is an hereditary $R$-order and $S$ is an indecomposable projective $\Lambda$-module. The following two conditions are equivalent for projective left $\Lambda$-modules $E$ and $F$ :

(i) $E$ and $F$ are $\Lambda$-isomorphic;

(ii) $E \otimes_{R} R_{p} \cong F \otimes_{R} R_{p}$ as $\Lambda_{p}$-modules and $\operatorname{Hom}_{\Lambda}(S, E)$ $\cong \mathrm{Hom}_{\Lambda}(S, F)$ are isomorphic as $R$-modules.

A canonical representation may be found in terms of the indecomposable module $S$ for any projective $\Lambda$-module $E$.

3. Concluding remarks. We may define a different for hereditary orders in a manner analogous to Deuring's definition for maximal orders [4]. This different localizes properly and has the desired multiplicative properties. We may then use the structure theory to compute the different explicity in terms of the different of maximal orders.

We have been able to apply our results to obtain new proofs of classical results on maximal orders as well as a few refinements of old theorems. 


\section{BIBLIOGRAPHY}

1. M. Auslander and O. Goldman, Maximal orders, Trans. Amer. Math. Soc. 97 (1960), 1-24.

2. - The Brauer group of a commutative ring, Trans. Amer. Math. Soc. 97 (1960), 367-409.

3. H. Cartan and S. Eilenberg, Homological algebra, Princeton Univ. Press, Princeton, N. J., 1956.

4. M. Deuring, Algebren, Springer, Berlin, 1935.

5. M. Rosen, Representations of twisted group rings, $\mathrm{Ph} . \mathrm{D}$. Thesis, Princeton University, Princeton, N. J., 1963.

6. O. F. G. Schilling, The theory of valuations, Math. Surveys No. 4, Amer. Math. Soc., Providence, R. I., 1950.

\section{Boston College}

